\title{
The Inverted Conductor-Backed Slotline - a Challenge to Antenna and Circuit Design
}

\author{
Jan Zehentner, Jan Machac, Jan Mrkvica, Carlo Tuzi \\ Czech Technical University, Faculty of Electrical Engineering, Technicka 2, 16627 Prague 6, Czech Republic \\ tel.: +420 224352273, fax: +420 224355865, E-mail: zehent@fel.cvut.cz
}

\begin{abstract}
Characteristics of modes on the conductorbacked slotline, the inverted conductor-backed slotline and on its modification in which the top dielectric layer with higher permittivity has a finite thickness are presented in this paper. Only the modified inverted conductor-backed slotline is able to transmit the dominant bound mode. The inverted and modified inverted conductor-backed slotlines are real candidates for the design of leaky wave antennas.
\end{abstract}

\section{INTRODUCTION}

The most important mode propagating on a planar transmission line is the dominant bound mode, since signals are transmitted by means of it. Beside this dominant bound mode, most planar transmission lines excite leaky modes. Generally speaking, leaky modes are undesirable, particularly in circuits. Exceptionally one of them was utilized in the directional coupler test. Leaky wave antennas are examples of practical applications of space leaky modes [1]. In this paper we present our most recent findings and contributions to an analysis of the conductor-backed slotline (CBSL), the inverted conductor-backed slotline (ICBSL) and to its modification (MICBSL).

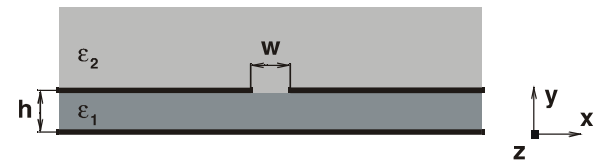

Fig. 1 Cross-section of the CBSL when $\varepsilon_{1}>\varepsilon_{2}$ and of the ICBSL when $\varepsilon_{1}<\varepsilon_{2}$. The coordinate system is shown.

A standard slotline (SL) transmits the pure dominant mode with even symmetry of the transversal component of the electric field within the slot. The first odd space leaky mode on the SL enabled the design of an antenna with a double-sided radiation pattern [1]. The requirement to radiate power only in the half space led us to an investigation of the CBSL, Fig. 1 when $\varepsilon_{1}>\varepsilon_{2}$. It turned out that this line is not suitable for signal transmission [2]. The line supports the dominant mode, which is unbound in the substrate and has a standing wave character laterally. This mode may propagate from zero frequency up to the cut-off frequency of the first mode leaking in the substrate. A real source of finite size excites the dominant mode due to reflections of the field directly radiated by the source from the substrate side walls [2]. Space leaky modes which also have strong leakage in the substrate occur on the CBSL with a sufficiently wide slot. The ICBSL and the MICBSL therefore show more promising features regarding signal transmission and radiation in space.

\section{INVERTED CONDUCTOR-BACKED SLOTLINE}

The cross-section of the ICBSL is shown in Fig. 1, when $\varepsilon_{1}<\varepsilon_{2}$. The name of this line comes from the inverted ratio of the first and second medium permittivities in comparison with the previous classical arrangement when $\varepsilon_{1}>\varepsilon_{2}$. The ICBSL possesses modes potentially able to leak power in the space above the line, since their phase constants are lower than $\mathrm{k}_{2}=\sqrt{ } \varepsilon_{\mathrm{r} 2} \mathrm{k}_{0}$. Moreover, the ICBSL supports a space leaky mode which does not simultaneously leak in the substrate where it is bound. The newly-revealed dominant leaky mode, with even symmetry of the transversal electric field component within the slot, possesses this character. Its dispersion characteristic is calculated without the contribution of any pole. The dispersion characteristic of this mode and the other even mode leaking simultaneously in the space and in the substrate are plotted in Fig. 2. The attenuation constant of the dominant leaky mode decreases to zero with decreasing frequency, and its cut-off is zero.

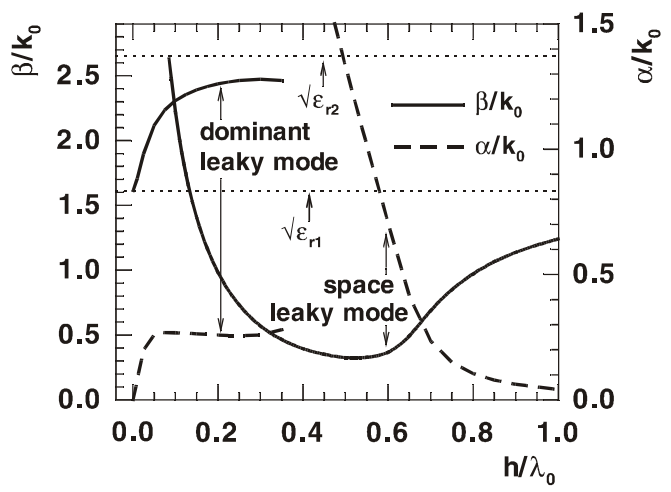

Fig. 2 Dispersion characteristics of even modes on the ICBSL with $\mathrm{w}=6 \mathrm{~mm}, \mathrm{~h}=6 \mathrm{~mm}, \varepsilon_{\mathrm{r} 2}=7$ and $\varepsilon_{\mathrm{r} 1}=2.6$.

The electric field distribution of the dominant mode evolves with the frequency increase from the quasi TEM mode, with the field uniformly distributed to infinity within the substrate, to the field bound to the slot in the substrate and leaking energy in the space shown in Fig. 3. The evolution of the dominant mode field in the substrate with increasing frequency is shown in Fig. 4.

According to the generalized condition of leakage [3] no mode leaking in the substrate is physical on the ICBSL 
since its phase constant should be lower than $\mathrm{k}_{\mathrm{TEM}}=\sqrt{ } \varepsilon_{\mathrm{r} 1} \mathrm{k}_{0}$, the phase constant of the TEM mode propagating in the parallel plate waveguide, and at the same time higher than $\mathrm{k}_{2}=\sqrt{ } \varepsilon_{\mathrm{r} 2} \mathrm{k}_{0}$. However, since $\varepsilon_{2}>\varepsilon_{1}$, this condition cannot be met.

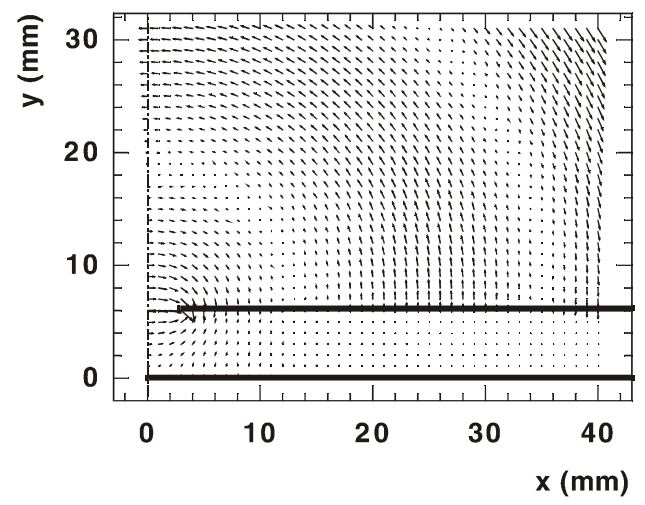

Fig. 3 Computed electric field distribution of the even dominant leaky mode on the transversal plane of the ICBSL from Fig. 2 at $\mathrm{h} / \lambda_{0}=0.1(5 \mathrm{GHz})$.

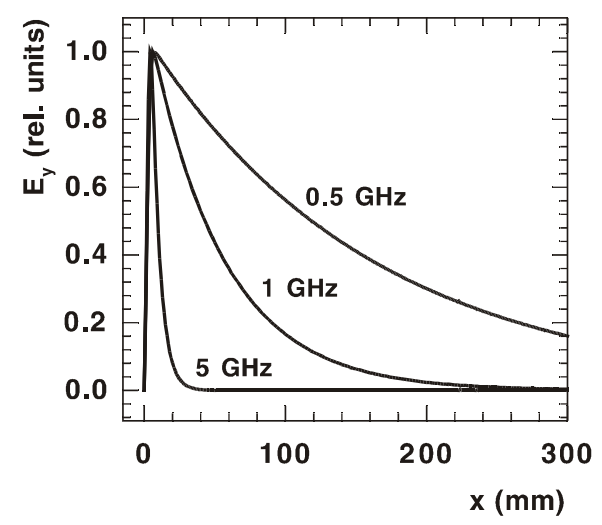

Fig. 4 Computed distribution of the $\mathrm{E}_{\mathrm{y}}$ field in the substrate perpendicular to the line axis, at the level $y=3 \mathrm{~mm}$ for the ICBSL from Fig. 2 at three frequencies. The fields are normalized to 1 .

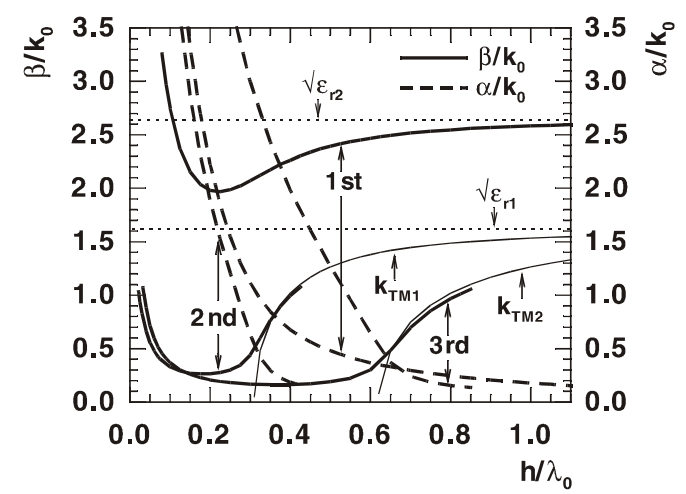

Fig. 5 Dispersion characteristics of odd space leaky modes on the ICBSL with $\mathrm{w}=6 \mathrm{~mm}, \mathrm{~h}=6 \mathrm{~mm}, \varepsilon_{\mathrm{r} 2}=7$ and $\varepsilon_{\mathrm{r} 1}=2.6$.

In addition, we identified the standard odd space leaky modes on the ICBSL. Their dispersion characteristics are shown in Fig. 5. The 1st characteristic was calculated without the contribution of any pole. It corresponds to the mode bound in the substrate, like the even dominant mode shown in Fig. 3, and leaks in the space. When calculating the characteristics marked 2 nd and $3 \mathrm{rd}$, the contribution of the pole which corresponds to the TEM parallel-plate mode in the substrate was accounted for. Consequently these two modes are physical up to the frequencies at which their characteristics cross the propagation constant of the $\mathrm{TM}_{1}$ substrate mode. Unlike the field of the even dominant leaky mode shown in Fig. 3 , the field of these two modes also grows laterally in the substrate, as shown in Fig. 6.

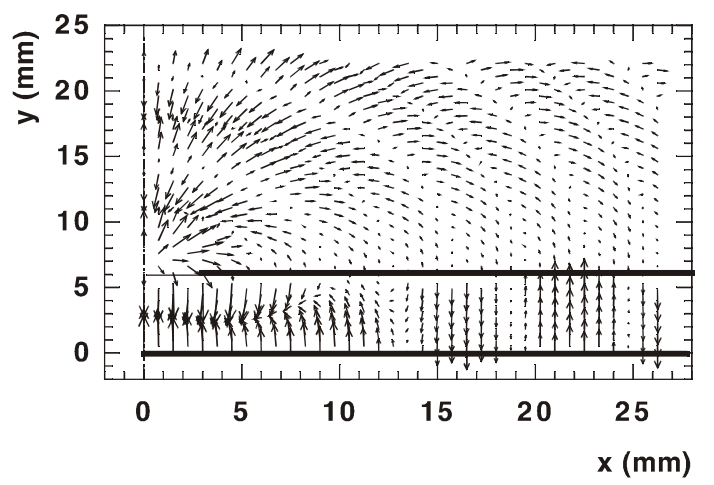

Fig. 6 Computed electric field of the odd space leaky mode corresponding to the 2 nd branch of the dispersion characteristic in Fig. 5 on the transversal plane of the ICBSL from Fig. 5 at $\mathrm{h} / \lambda_{0}=0.35(17.5 \mathrm{GHz})$.

\section{MODIFIED INVERTED CONDUCTOR-BACKED SLOTLINE}

In the ICBSL the medium with permittivity $\varepsilon_{2}>\varepsilon_{1}$ surrounding the line draws the field up through the slot in the open space. This medium must have a finite thickness in real circuits and radiating elements. So we will now deal with the modified ICBSL with the cross-section shown in Fig. 7. The dispersion characteristics of

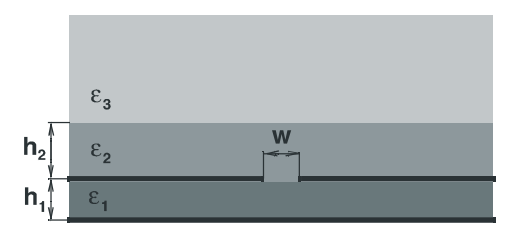

Fig. 7 Cross-section of the modified ICBSL when $\varepsilon_{3}<\varepsilon_{1}<\varepsilon_{2}$.

the even modes are plotted in Fig. 8. The dominant bound mode, bound in the $1 \mathrm{st}$, 2nd and 3rd dielectric, propagates from zero frequency. The field distribution of this dominant bound mode in the cross-sectional plane of the MICBSL is plotted in Fig. 9. This mode is physical up to the point at which its dispersion characteristic touches the characteristic of the $\mathrm{TM}_{0}$ mode. At higher frequencies it proceeds as the nonphysical solution of the dispersion equation. The characteristic of the mode leaking in the top dielectric slab (1st leaky, Fig. 8) splits off from this nonphysical solution, and becomes physical when its phase constant falls below $\mathrm{k}_{\mathrm{TM} 0}$. The second branch of the 1 st leaky mode characteristic splits off from the nonphysical solution at a fairly high frequency, and is nonphysical for the line from Fig. 8, as its phase constant is higher than $\mathrm{k}_{\mathrm{TM} 0}$. The second leaky mode sets up above the cut-off of the $\mathrm{TE}_{1}$ mode. This mode represents the leakage of energy simultaneously into the $\mathrm{TM}_{0}$ and $\mathrm{TE}_{1}$ surface modes of the upper dielectric layer, and is physical in the frequency bands at which its phase 
constant is lower than $\mathrm{k}_{\mathrm{TE} 1}$ and at the same time higher than $\mathrm{k}_{\mathrm{TEM}}=\sqrt{ } \varepsilon_{\mathrm{r} 1} \cdot \mathrm{k}_{0}$. For the line from Fig. 8, this happens when $\mathrm{h} / \lambda_{0}=0.13-0.215$. Due to the rather high leaky constant it decreases rapidly with distance from the point of excitation. Lowering of the top slab thickness results in the characteristics shown in Fig. 10.

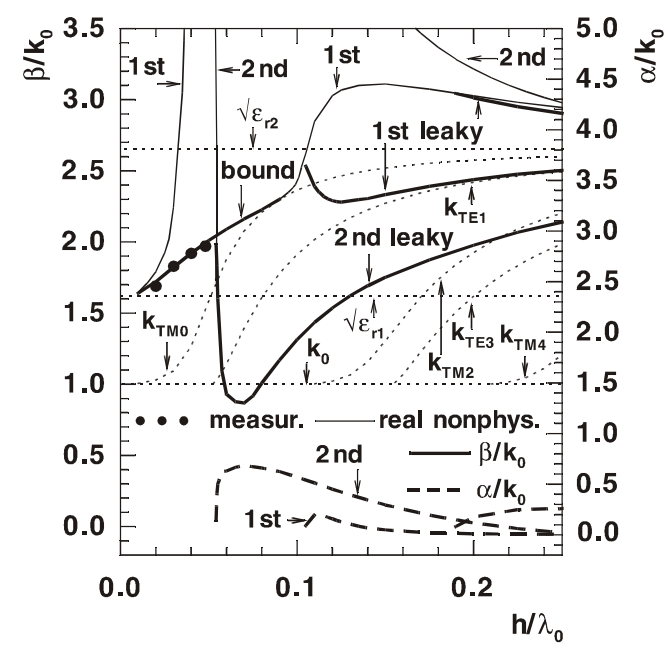

Fig. 8 Dispersion characteristics of even modes on the MICBSL with $\mathrm{w}=6 \mathrm{~mm}, \mathrm{~h}_{1}=6 \mathrm{~mm}, \mathrm{~h}_{2}=12 \mathrm{~mm}, \varepsilon_{\mathrm{r} 1}=2.6, \varepsilon_{\mathrm{r} 2}=7$ and $\varepsilon_{\mathrm{r} 3}=1$.

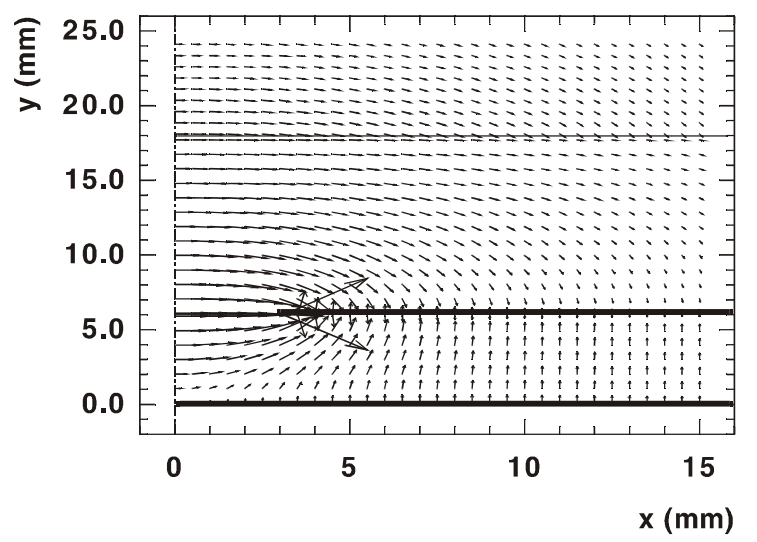

Fig. 9 Computed electric field of the even dominant bound mode on the MICBSL from Fig. 8 at the normalized frequency $\mathrm{h} / \lambda_{0}=0.04$.

The patterns of the two branches of the 1st leaky mode dispersion characteristic shown in Fig. 8 are now similar to those described in [4] for the 1st leaky mode on the CBSL with a wide slot. The split-off points of the two branches of the 1st leaky mode characteristic, not shown in Fig 10, merge and become identical when the thickness of the upper dielectric decreases, and finally we get the ambiguous dispersion characteristic with two significantly different propagation constants. The upper branch is physical up to the frequency at which $\beta$ becomes lower than $\mathrm{k}_{\mathrm{TM} 0}$. Additionally these two branches correspond to the modes with different field distributions which differ more and more with increasing frequency, as documented in Fig. 11.

We verified the excitation of the dominant bound mode on the MICBSL. The plexiglass substrate has $\varepsilon_{\mathrm{r} 1}=2.6$ and the top glass slab has $\varepsilon_{\mathrm{r} 2}=7$. A movable probe

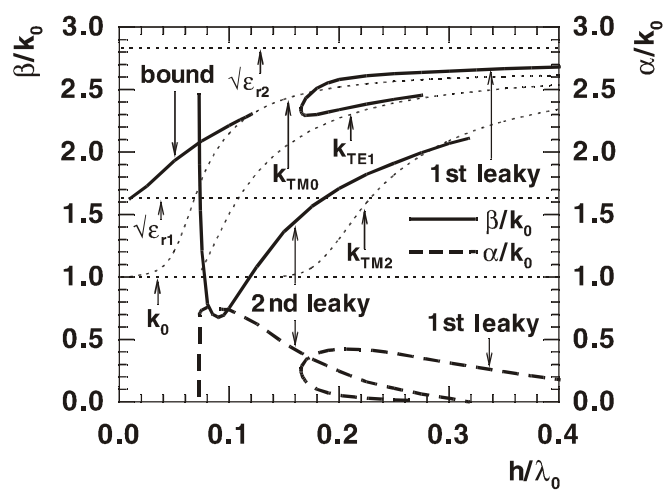

Fig. 10 Dispersion characteristics of even modes on the MICBSL with w $=6 \mathrm{~mm}, \mathrm{~h}_{1}=6 \mathrm{~mm}, \mathrm{~h}_{2}=9 \mathrm{~mm}, \varepsilon_{\mathrm{r} 1}=2.6, \varepsilon_{\mathrm{r} 2}=7$ and $\varepsilon_{\mathrm{r} 3}=1$.

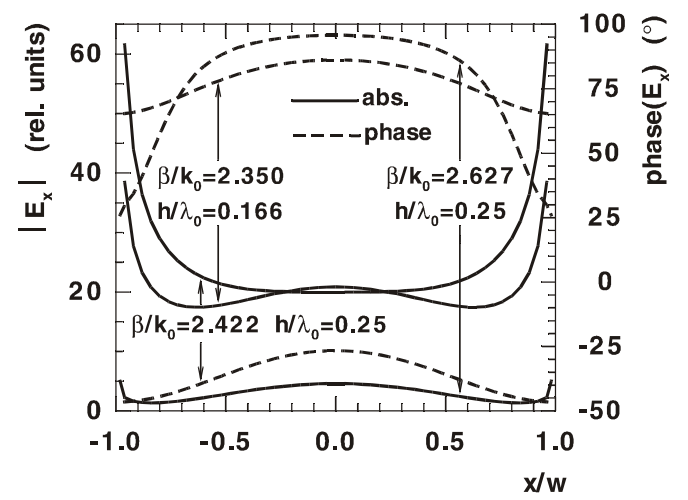

Fig. 11 The calculated distribution of the $E_{x}$ field of the 1 st leaky mode within the slot of the MICBSL from Fig. 8 at $\mathrm{h} / \lambda_{0}=0.166$, the very left point of the ambiguous characteristic, and at its two points when $\mathrm{h} / \lambda_{0}=0.25$.

measured the wavelength along the line axis on the top side of the glass. The results at four frequencies are marked in Fig. 8. The measured patterns, not shown here, confirmed unattenuated propagation of the dominant mode. The same probe, movable transversally on the top surface of the glass, recorded the vertical electric field component shown in Fig. 12 and compared here with the calculated distribution. The measured record is affected by the parasitic standing wave, when $\mathrm{x}$ is greater than 120 $\mathrm{mm}$, raised due to the reflections from the substrate edges.

The presence of the second dielectric gives rise to further leaky modes linked to leakage in the 1st and 2nd dielectric layer. MICBSL therefore offers a wide choice of dispersion characteristics in dependence on the size and permittivities of the used dielectrics. A deeper investigation into them is now in progress. When the top surface of the second dielectric is covered by metal the MICBSL is suitable for the design of circuit elements, eg. phase shifters. 


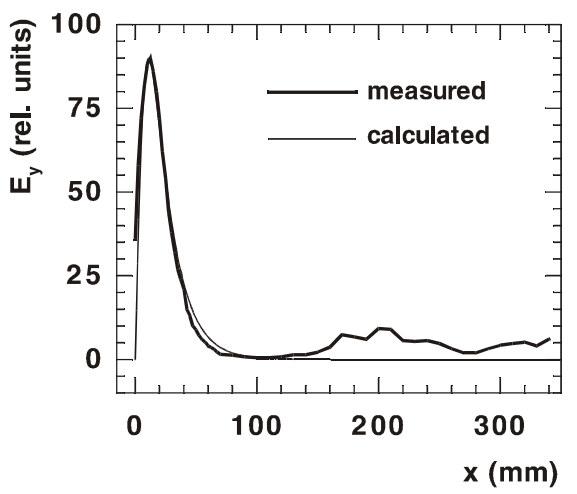

Fig. 12 Vertical electric field component $E_{y}$ measured and calculated on the top surface of the glass slab of the MICBSL from Fig. 8 at h/ $\lambda_{0}=0.04(2 \mathrm{GHz})$.

Fig. 13 demonstrates the capability of the MICBSL to leak in the half space. In addition to the odd dominant bound mode there are also two characteristics of the odd space leaky mode. The mode corresponding to the characteristic denoted " 2 " in Fig. 13 represents the leakage in the space, and only in the top dielectric slab, because the characteristic was calculated with the contribution of the pole linked to the upper dielectric layer mode $\mathrm{TM}_{0}$. This mode is nonphysical, since the characteristic lies below $\sqrt{ } \varepsilon_{\mathrm{rl}}$, the normalized phase constant of the TEM mode in the bottom substrate. The mode with the characteristic denoted " 1 " in Fig. 13 leaks in the space and simultaneously in the bottom and upper substrates. This is because the pole of the TEM mode in the bottom substrate and the pole linked to the upper dielectric layer mode $\mathrm{TM}_{0}$ were both taken into account. This mode is physical in the frequency band $\mathrm{h} / \lambda_{0}=0.02-$ 0.14 , in which its normalized phase constant is lower than one. The field distribution of this mode on the crosssectional plane of the MICBSL from Fig. 13 is plotted in Fig. 14. The field amplitude in both dielectric slabs increases, indicating leakage into these dielectrics.

\section{CONCLUSION}

Characteristics of the most important modes on the ICBSL and on the MICBSL are presented. To the authors' knowledge, most of these are new findings. They enable easier choice of the line cross-section and dielectric parameters with respect to the requirements laid on the line function. The existence of the most questionable modes was checked by field measurements. The experimental and theoretical results agree well. An open MICBSL is a suitable candidate for the design of a leaky wave antenna radiating only to one side of the substrate. On the other hand, a MICBSL covered on the top by metallization will enable the construction of new circuit elements utilizing propagation of even or odd dominant bound modes. MICBSL provides an opportunity to analyze the behaviour of the line in more detail and to reveal unsuspected dependences on many factors not accessible on CBSL or SL itself. The concept of the MICBSL can also be applied to the inverted conductor-backed coplanar waveguide.

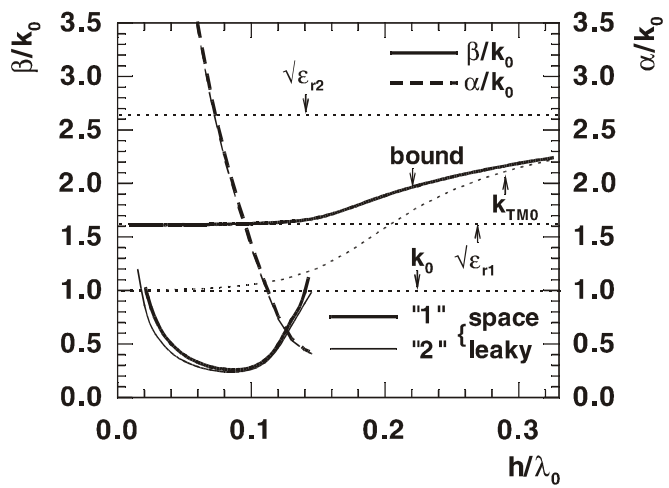

Fig. 13 Dispersion characteristics of the odd space modes on the MICBSL with $\mathrm{w}=12 \mathrm{~mm}, \mathrm{~h}_{1}=6 \mathrm{~mm}, \mathrm{~h}_{2}=3 \mathrm{~mm}, \varepsilon_{\mathrm{rl}}=2.6$, $\varepsilon_{\mathrm{r} 2}=7$ and $\varepsilon_{\mathrm{r} 3}=1$.

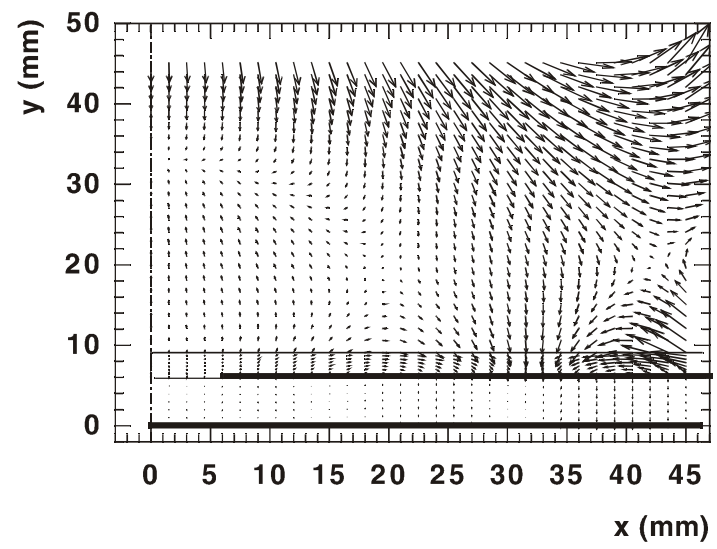

Fig. 14 Computed electric field of the odd space leaky mode corresponding to the characteristic " 1 " from Fig. 13 at $\mathrm{h} / \lambda_{0}=0.14(7 \mathrm{GHz})$.

\section{ACKNOWLEDGEMENT}

This work has been supported by the Grant Agency of the Czech Republic under project 102/03/0449 „New circuit devices for communication technology" and by the research program J04/98 212300014 „Information and Communication Technology".

\section{REFERENCES}

[1] J.-W. Sheen, Y.-D. Lin: Propagation Characteristics of the Slotline First Higher Order Mode, IEEE Trans. Microwave Theory Tech., vol. MTT-46, 1998, No. 11, pp. 1774-1781.

[2] J. Zehentner, J. Machac, J. Mrkvica, C. Tuzi: Modes on the standard and inverted conductor-backed slotline. 2003 IEEE MTT-S Int. Microwave Symp. Digest, vol 2, pp. $677-$ 680, June 2003.

[3] C. Di Nallo, F. Mesa, D. R. Jackson: Excitation of leaky modes on multilayer stripline structures. IEEE Trans. Microwave Theory and Tech., vol. MTT-46, no. 8, pp. 1062-1071, August 1998.

[4] J. Zehentner, J. Machac, J. Mrkvica: Even and odd modes on a conductor-backed slotline, Proc. of 32nd European Microwave Conference, vol. 2, pp. 609-612, Sept. 2002. 